\title{
Epidemiología del linfoma con compromiso de cabeza y cuello en el Centro Asistencial Sótero del Río
}

\author{
Epidemiology of lymphoma of the head and neck in the \\ Centro Asistencial Sótero del Río
}

Raimundo García M¹, Roberto Fernández G², Rubén González E³.

\begin{abstract}
RESUMEN
Introducción: Los linfomas son la neoplasia hematológica más frecuente del adulto, ocupando el tercer lugar de las neoplasias de cabeza y cuello (CyC). Se distinguen dos tipos, el linfoma de Hodgkin (LH) y el linfoma no Hodgkin (LNH), constituyendo dos entidades clínicas separadas.

Objetivo: Evaluar las manifestaciones de los linfomas en la región de CyC, analizando las diferencias de presentación y comportamiento entre en LH y el LNH.

Material y método: Estudio retrospectivo de todos los casos de linfomas de CyC en el Centro Asistencial Dr. Sótero del Río (CASR) entre los años 2005 y 2009, documentando específicamente presentación clínica, factores de riesgo, el tipo de linfoma, diagnóstico histológico, localización anatómica, etapificación al momento del diagnóstico, tratamiento administrado, sobrevida.

Resultados: Se incluyeron 80 casos de pacientes con linfoma de CyC en el estudio, 16 (20\%) con LH y 64 (80\%) con LNH. Se encontraron diferencias estadísticamente significativas entre ambos para la edad de diagnóstico, el compromiso extranodal, compromiso de mediastino, presencia de síntomas $B$ y sobrevida.

Conclusión: En el CASR la presentación de los linfomas es similar a la reportada mundialmente con diferencias similares entre $\mathrm{LH}$ y $\mathrm{LNH}$.

Palabras clave: Linfoma, epidemiología, Chile, otorrinolaringología.
\end{abstract}

\begin{abstract}
Introduction: Lymphomas are the most common hematologic neoplasia in the adult and the third most common neoplasias of the head and neck (CyC). There are two types of lymphoma: Hodgkin and non-Hodgkin lymphomas, which constitute two unique clinical entities.

Aim: To evaluate the clinical manifestations of lymphomas in the CyC region, analizing the diferences between the clinical presentation and behavior of $\mathrm{LH}$ and $\mathrm{LNH}$.

Material and method: This is an retrospective study of all the cases of lymphoma of CyC in the Centro Asistencial Dr. Sótero del Río (CASR) between the years 2005 and
\end{abstract}

\footnotetext{
${ }^{1}$ Médico Cirujano. Pontificia Universidad Católica de Chile.

${ }^{2}$ Médico. Servicio de Otorrinolaringología, Hospital Sótero del Río.

${ }^{3}$ Médico. Departamento de Otorrinolaringología, Pontificia Universidad Católica de Chile.
} 
2009, describing specifically the clinical presentation, risk factors, type of lymphoma, histologic diagnosis, anatomical localization, etapification at diagnosis, treatment received and survival.

Results: Eighty cases of lymphoma of the CyC were included, 16 patients (20\%) with $\mathrm{LH}$ and 64 patients (80\%) with LNH. We found statistically significant differences between both groups for their age at diagnosis, extranodal disease, mediastinal compromise, presence of "B" symptoms and survival.

Conclusion: in the CASR the clinical presentation of lymphomas is similar to that reported in the international literature, maintaining the unique characteristics for $\mathrm{LH}$ and LNH.

Key words: Lymphoma, epidemiology, Chile, otorrinolaryngology.

\section{INTRODUCCIÓN}

Los linfomas son la neoplasia hematológica más frecuente del adulto ${ }^{1}, y$ representan aproximadamente el $5 \%$ de las neoplasias de cabeza y cuello $(\mathrm{CyC})^{2}$. Corresponden a una proliferación de células neoplásicas del tejido linfoide en los ganglios linfáticos (enfermedad nodal) o fuera de ellos (extranodal).

Se distinguen dos tipos, el linfoma de Hodgkin (LH) y el linfoma no Hodgkin (LNH), constituyendo dos entidades clínicas separadas.

En Chile, la incidencia de estas enfermedades es de 1,5 y 5,6 por 100.000 habitantes para el LH y $\mathrm{LNH}$, respectivamente, cifras similares a las observadas internacionalmente ${ }^{3}$. El último tiempo se ha observado un aumento en la incidencia de los linfomas debido al surgimiento de condiciones inmunosupresoras (VIH, trasplantes), infecciones crónicas (Epstein Barr, Helycobacter pylori, HTLV1) y el envejecimiento de la población, lo que podría cambiar la epidemiología de los linfomas ${ }^{4}$.

El LNH, en comparación con el LH, presenta más frecuentemente invasión local y desarrollo en sitios extranodales, se encuentra más diseminado al momento del diagnóstico y tiene peor pronósti$\mathrm{co}^{5}$. Ambas enfermedades se presentan en una alta proporción como una adenopatía silente o masa en estudio en CyC siendo derivadas al otorrinolaringólogo y constituyendo un desafío diagnóstico.

En 1988 se creó en Chile el Programa de Cáncer del Adulto (PANDA), dependiente del Ministerio de Salud, con el objetivo de financiar las drogas para el tratamiento de enfermedades neoplásicas con esquemas de reconocida eficacia a nivel mundial. La atención se realiza en 18 centros acreditados a lo largo de todo el país, dentro de los que se encuentra el Centro Asistencial Dr. Sótero del Río (CASR).

Desde el año 2005, con la incorporación de los linfomas al programa de Garantías Explícitas en Salud (GES), todos los pacientes con sospecha diagnóstica de linfoma son derivados a un centro PANDA con el fin de confirmar el diagnóstico e iniciar tratamiento, llevando un registro de los pacientes ingresados al programa.

El objetivo de este estudio es evaluar las manifestaciones de los linfomas en la región de CyC, analizando las diferencias de presentación y comportamiento entre en LH y el LNH.

\section{MATERIAL Y MÉTODO}

Se realizó una revisión retrospectiva de todos los casos de linfomas de CyC en el CASR entre los años 2005 y 2009. Se documentó específicamente presentación clínica, factores de riesgo, el tipo de linfoma, localización anatómica, etapificación al momento del diagnóstico (utilizando la clasificación de Ann Arbor), tratamiento administrado y sobrevida.

Para las variables continuas se reportaron medias y desviaciones estándar; excepto para la sobrevida que se usaron medianas y rango. Para las variables categóricas se realizaron tablas de frecuencias, con las respectivas frecuencias y porcentajes. Para averiguar si existía asociación entre variables categóricas se realizaron tablas de contingencia y se calculó el valor $p$ a partir del test chi cuadrado de Pearson, el estadístico de Fisher 0 asociación lineal por lineal, según correspondiera. 
Para establecer asociaciones entre una variable continua y otra categórica se realizó una prueba de normalidad y luego la prueba no paramétrica de Mann-Whitney. Los tiempos de sobrevida fueron calculados por el método de Kaplan Meier. Se consideró significativo valores $p$ inferiores a 0,05. Para los análisis se usó el programa estadístico SPSS ${ }^{\circledR}$ 17.0.

\section{RESULTADOS}

Se encontraron 346 casos de linfoma en el CASR entre los años 2005 y 2009. De éstos, hubo 80 casos que presentaban compromiso de CyC, siendo todos ellos incluidos en el estudio. De éstos, 16 $(20 \%)$ correspondían a LH y $64(80 \%)$ a LNH.

Edad. La edad media al diagnóstico fue de 28,9 y 56,8 en los pacientes con LH y LNH, respectivamente. Esta diferencia fue significativa $(p<0,001)$ (Figura 1).

Género. El $81,3 \%$ de los pacientes era de sexo masculino en LH y el $57,8 \%$ en LNH ( $p=0,083)$. Sin diferencia estadística.

Compromiso nodal. El $100 \%$ de los pacientes con LH y el $81,3 \%$ de los pacientes con LNH presentaron compromiso nodal ( $p=0,111)$.

Compromiso extranodal. Ningún paciente con LH presentó compromiso extranodal, mientras que el 37,5\% de los pacientes con LNH sí lo presentó. Esta diferencia fue significativa $(p=0,002)$. La distribución del compromiso extranodal se puede observar en la Figura 2, siendo los lugares más frecuentes las amígdalas, el seno maxilar y la región ocular.

Compromiso extra CyC. De los pacientes con LH, $62,5 \%$ presentaron compromiso linfático en otra región anatómica diferente a la región de CyC. Esta situación ocurrió en $36 \%(p=0,054)$ de los pacientes con LNH. La ubicación más frecuente de las adenopatías extra CyC fueron en el mediastino (7), grupos supraclaviculares (3) y pulmonares (3) en LH; y los grupos axilares (14), inguinales (7) y el mediastino (7) en los LNH (Tabla 1).

Compromiso de médula ósea (MO). Doce coma cinco por ciento de los pacientes con LH presentó compromiso de MO comparado con $9,4 \%$ de los pacientes con LNH ( $p=0,657$ ).

Factores de riesgo. De los pacientes con LH, el $31,2 \%$ y el $37,5 \%$ presentaban antecedentes de tabaquismo y consumo de alcohol, respectivamente. En los pacientes con LNH, el $25 \%$ presentaba antecedentes de tabaquismo ( $p=0,532)$ y $39 \%$ de consumo de alcohol $(p=0,532)$.

Presentación clínica. Setenta y cinco por ciento y $70 \%$ de los pacientes con LH y LNH, respectivamente, presentó aumento de volumen o masa como parte de su cuadro inicial. En cuanto a los síntomas constitucio-

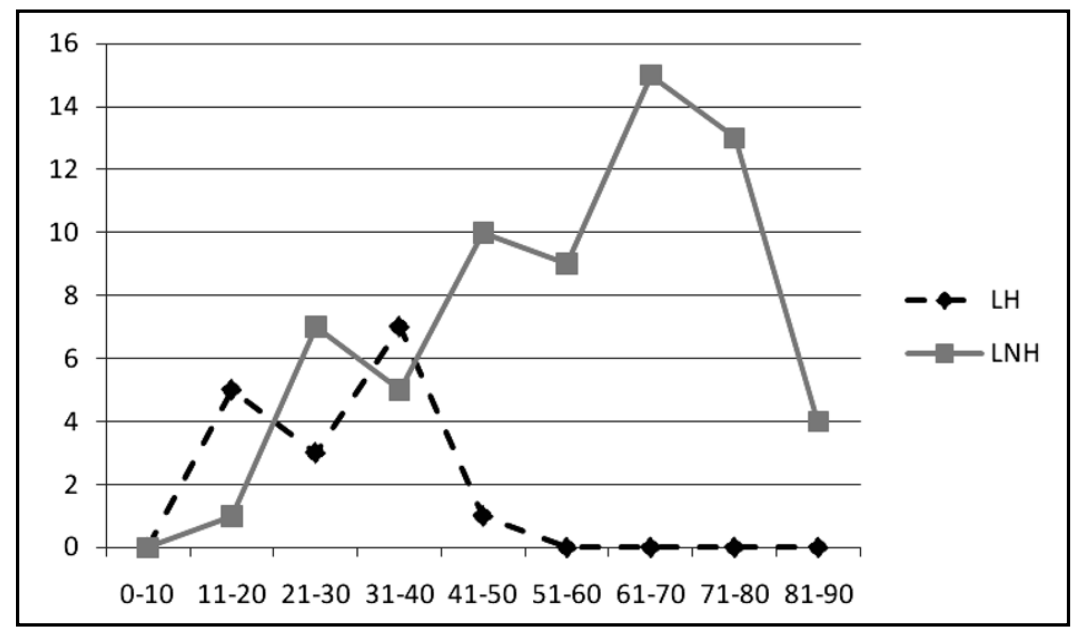

Figura 1. Distribución por grupo etario del LH y LNH. Gráfico que muestras las diferencias de acuerdo a la edad de presentación entre el LH y LNH $(p<0,001)$. 


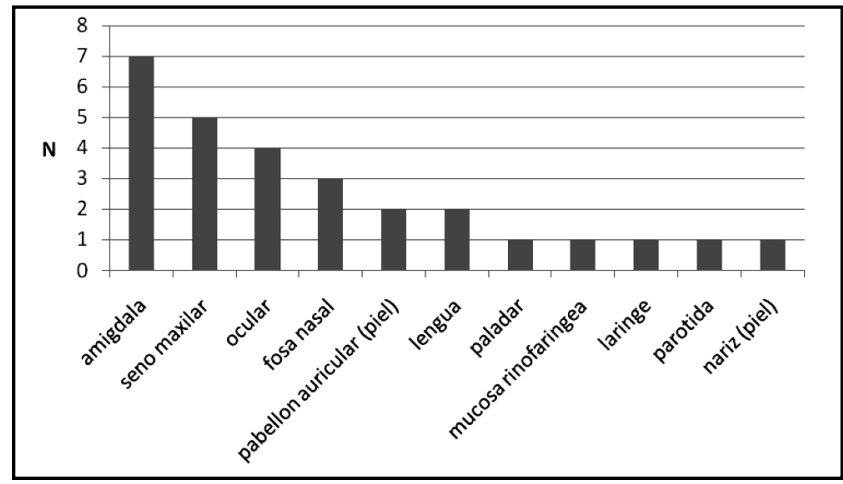

Figura 2. Distribución del compromiso extranodal en el LNH de CyC.

Tabla 1. Compromiso de grupos linfáticos en territorios extra CyC en LH y LNH

\begin{tabular}{|llll|}
\hline Grupo linfático comprometido & $\begin{array}{l}\text { LH }(16) \\
\mathrm{n}(\%)\end{array}$ & $\begin{array}{l}\text { LNH }(64) \\
\mathrm{n}(\%)\end{array}$ & $\mathrm{p}$ \\
\hline Supraclavicular & $3(18,8)$ & $5(7,8)$ & 0,194 \\
Axilar & $2(12,5)$ & $14(21,9)$ & 0,505 \\
Mediastino & $7(43,8)$ & $7(11)$ & $0,005^{\star}$ \\
Pulmonar & $3(18,8)$ & $2(3)$ & 0,052 \\
Retroperitoneo & $2(12,5)$ & $6(9,3)$ & 0,657 \\
Mesenterio & $1(6,3)$ & $4(6,3)$ & 1 \\
Lumboaórtico & $1(6,3)$ & $2(3)$ & 0,493 \\
Tronco celíaco & $1(6,3)$ & $1(1,6)$ & 0,362 \\
Inguinal & 0 & $7(11)$ & 0,334 \\
\hline Total & $10(62,5)$ & $23(36 \%)$ & 0,054 \\
\hline
\end{tabular}

* Diferencia estadísticamente significativa.

nales (síntomas B), 31\% de los pacientes con $\mathrm{LH}$ presentó 203 síntomas B. El 37\% presentó baja de peso, $31 \%$ diaforesis nocturna y $25 \%$ fiebre. De los pacientes con $\mathrm{LNH}, 3 \%$ presentó 203 síntomas $\mathrm{B}$ ( $\mathrm{p}$ $=0,006)$, siendo el más frecuente la baja de peso con $18,8 \%(p=0,098)$, luego la diaforesis nocturna con $7,8 \%(p=0,019)$ y finalmente la fiebre con $6,2 \%(p$ $=0,04)$. Cuatro $(25 \%)$ pacientes con LH presentaron algún síntoma local, éstos fueron disnea (1), tos (1), odinofagia (1) y parestesias (1). De los pacientes con $\mathrm{LNH}$, trece $(20 \%)$ presentaron síntomas locales $(p$ $=0,79$ ) siendo los más frecuentes disfagia (5), disnea (3), odinofagia (2) y tos (2). Un paciente con LNH se presentó como un síndrome de vena cava superior.
Etapificación. La etapa en que se encontraban los pacientes al momento del diagnóstico se muestra en la Tabla 2. No hubo diferencias estadísticas en cuanto a la distribución por grupos.

Tratamiento. El tratamiento utilizado en LH y LNH se puede observar en la Tabla 3.

Sobrevida. El $100 \%$ de los pacientes con LH estaba vivo, comparado con el $40,6 \%$ de los pacientes con LNH que habían fallecido hasta el momento del estudio $(p=0,002)$. El seguimiento promedio de fue de 44 meses en los casos de LH y de 29 meses en los LNH. El seguimiento y la sobrevida se muestran graficados en la Figura 3. 
Tabla 2. Etapificación de la enfermedad al momento del diagnóstico en LH y LNH

\begin{tabular}{|lcc|}
\hline & Linfoma de Hodgkin & Linfoma no Hodgkin \\
\hline Etapa I & $13,3 \%$ & $17 \%$ \\
Etapa II & $46,7 \%$ & $29,2 \%$ \\
Etapa III & $33,3 \%$ & $39 \%$ \\
Etapa IV & $6,7 \%$ & $14,6 \%$ \\
\hline
\end{tabular}

Tabla 3. Esquema de tratamiento administrado en LH y LNH

\begin{tabular}{|lll|}
\hline & LH & LNH \\
\hline Quimioterapia (esquema; N; \%) & ABDV; $11 ; 68,8 \%$ & CHOP'; $38 ; 59,3 \%$ \\
& & RCHOP $; 7 ; 11 \%$ \\
& & Clorambucil; $4 ; 6,25 \%$ \\
& & ESHAP\$; $2 ; 3,1 \%$ \\
Quimioterapia + radioterapia (N; \%) & $5 ; 31,2 \%$ & COPII; $1 ; 1,6 \%$ \\
Radioterapia única (N;\%) & - & $11 ; 17,2 \%$ \\
& & $1 ; 1,6 \%$ \\
\hline
\end{tabular}

*ABVD: Adriamicina, Bleomicina, Vinplastina, Dacarbizina; ${ }^{\dagger} \mathrm{CHOP}:$ COP + doxorrubina; `RCHOP: CHOP + rituximab; \$ESHAP: Etopósiodo, Metilprednisolona, Cytarabina, Cisplatino; "COP: Ciclofosfamida, vincristina y prednisolona.

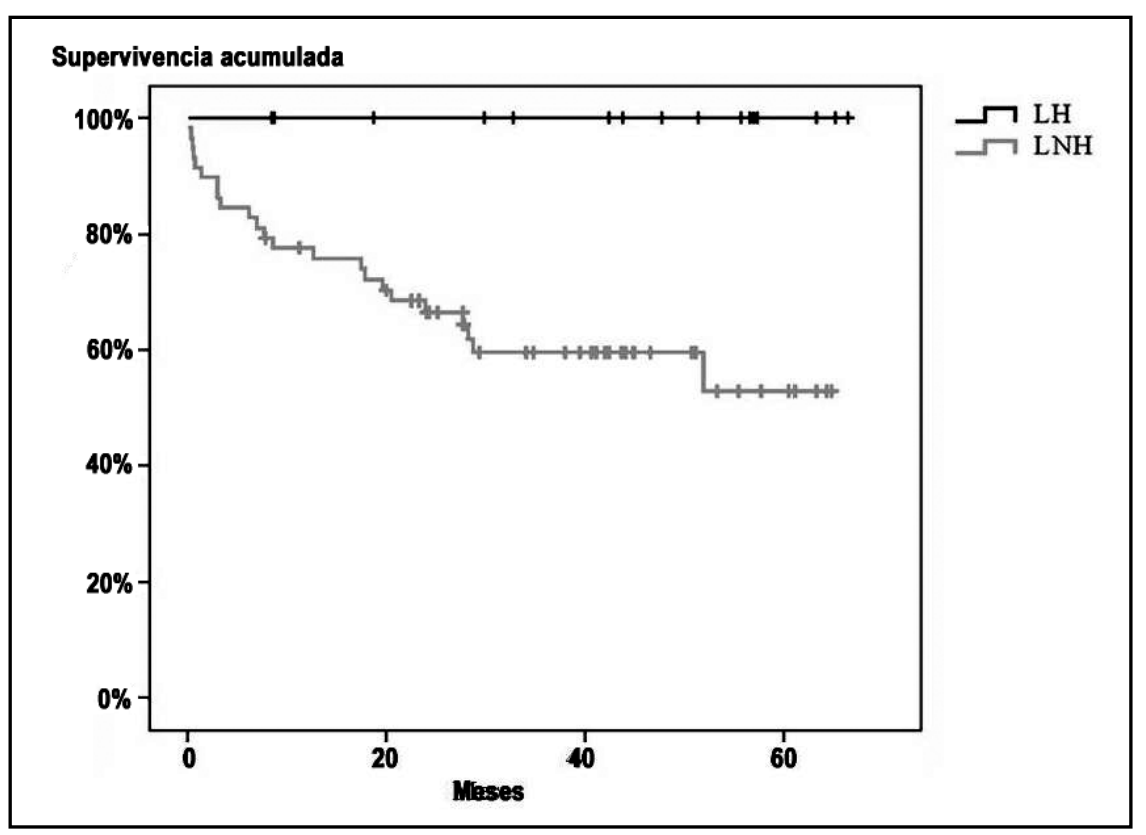

Figura 3. Supervivencia (sobrevida y seguimiento) de los pacientes con LH y LNH (método Kaplan Meier). 


\section{DISCUSIÓN}

Los linfomas que comprometen la CyC en algún momento de su evolución representan el $23 \%$ de todos los linfomas del CASR. De éstos, el $90 \%$ tiene su primera manifestación clínica (debut de enfermedad) en la CyC. De las manifestaciones iniciales la más frecuente es la adenopatía cervical (68\%), que corresponde al $94 \%$ en LH y al $53 \%$ en LNH. Luego siguen las adenopatías extra CyC (axilar, mediastino) y finalmente, sitios extranodales (sólo en LNH).

Es de gran importancia que estas características de la presentación inicial del linfoma (alta prevalencia en CyC especialmente adenopatía cervical) sea conocida por el otorrinolaringólogo para incorporarlo al diagnóstico diferencial de masas y adenopatía de CyC, manteniendo un alto índice de sospecha en estos casos y lograr un diagnóstico oportuno de esta patología.

Complementando lo anterior, es sabido que la CyC es el segundo sitio anatómico más frecuente para la aparición de linfomas extranodales ${ }^{6}$, lo que ocurrió en el $33 \%$ de los pacientes con LNH en su debut y en $44 \%$ durante toda su evolución. Esto ilustra que se debe considerar al linfoma en el diagnóstico diferencial de cualquier lesión de CyC, incluyendo la cavidad nasal, orofaríngea, laringe y los senos paranasales ${ }^{6,7}$.

En cuanto a la edad de presentación, en nuestro trabajo se observan las mismas diferencias de edad entre el LH y el LNH que las reportadas en la literatura. La edad media observada en el LNH fue de 57 años (rango 15-89) con una incidencia en aumento según la edad, comparable con lo descrito previamente ${ }^{5,8}$. El promedio de edad de los pacientes con LH fue de 29 años con un rango entre 16 y 46 años sin observarse el peak bimodal a los 15-35 años y a los 50 años descrito clásicamente (Figura 1) ) $^{3,9}$. En un estudio chileno por Cabrera y cols. que incluyó 682 pacientes con LH se encontró un promedio de 37 años de edad con un rango de 15-84 años ${ }^{9}$. Urquhart y cols ${ }^{5}$. estudiaron 76 pacientes con $\mathrm{LH}$ de CyC encontrando un promedio de edad de 27,7; y en un estudio de lyengar y cols ${ }^{10}$. que incluyó 39 pacientes con LH de CyC se encontró un promedio de edad de 31,5 años con un rango entre 6 y 88 años. Consideran- do además nuestros datos, se observa una tendencia a que el LH de CyC ocurra en pacientes más jóvenes comparado con el LH en general, lo que debe tomarse en cuenta ante la sospecha diagnóstica. Nuestro rango etario más limitado podría explicarse por el menor número de pacientes.

El sexo masculino predominó en ambos casos (no significativo) lo que concuerda con lo reportado previamente ${ }^{8,9}$. No existe aún alguna explicación para esta tendencia en los linfomas.

En cuanto a la sintomatología, encontramos que la presencia de 20 más síntomas constitucionales (síntomas $B$ ) constituye un buen predictor diagnóstico de LH versus LNH ( $p=0,006)$, especialmente la sudoración nocturna $(p=0,019)$ y la fiebre $(p=0,04)$. Por otro lado, no encontramos diferencias significativas en la presencia de síntomas locales que nos permitieran discriminar entre ambas enfermedades.

Los hábitos de vida, especialmente el consumo de alcohol y el tabaquismo son conductas prevalentes consideradas factores de riesgo para varios tipos de cáncer. En cuando a la relación con los linfomas, el consumo de alcohol protegería del LH y LNH, mientras que para el tabaquismo hay datos contradictorios sin establecerse una tendencia definitiva ${ }^{11-15}$. En nuestro trabajo no estudiamos estos datos comparados con la población general, pero al analizar la prevalencia de estos hábitos entre ambos tipos de linfomas, no encontramos diferencias significativas, lo que concuerda con la literatura. Es conocido que el tabaquismo actúa como factor pronóstico independiente en los casos de $\mathrm{LNH}^{16}$, lo que en nuestro estudio no se pudo observar con significancia estadística aunque sí se observó la tendencia.

El LH constituye una de las neoplasias más curables hoy en día, con 60\%-70\% de curación, lo que es dependiente de la etapa al momento del diagnóstico $0^{9,17}$. En nuestra casuística, los 2 pacientes en que fracasó el tratamiento se encontraban en etapa avanzada, que está de acorde a lo reportado, pero que no resultó ser estadísticamente significativo, probablemente por el tamaño muestral.

En el caso del LNH, si bien se ha demostrado que el pronóstico depende principalmente de la 
histopatología, existen otros factores pronósticos como la edad, enfermedad extranodal y etapificación que también influirían ${ }^{2,18}$. En el último tiempo se ha visto que las etapas iniciales del LNH en CyC tienen una buena respuesta a los nuevos tratamientos orientados según el tipo histológico, alcanzando una sobrevida a los 5 años del 83\%. Esto ilustra la importancia del diagnóstico temprano y la elección de la terapia adecuada según el tipo de linfoma ${ }^{19}$. En nuestro trabajo no encontramos diferencias significativas en estos últimos factores con respecto a la sobrevida en el LNH, aunque creemos que es debido al tamaño de la muestra. Tampoco encontramos diferencias estadísticamente significativas en el compromiso de médula ósea (factor pronóstico de la enfermedad y que influye en su etapificación) entre ambos tipos de linfoma y como factor pronóstico en el LNH.

Nuestro seguimiento promedio fue de 44 meses para LH y de 29 meses para LNH, observándose una mortalidad de $0 \%$ y $40 \%$ respectivamente ( $p=0,002)$. Si bien llama la atención la baja mortalidad reportada en ambos tipos de linfoma, esto se explica por el tiempo de seguimiento insuficiente para pesquisar todas las recidivas que se esperarían, y la mayor frecuencia de etapas iniciales de nuestro estudio.

Los linfomas son enfermedades con incidencia y mortalidad en aumento en Chile y el mundo. Presentan una tasa de respuesta a tratamiento dependiente de la etapa al momento de diagnóstico, especialmente el LH. Considerando el alto porcentaje de casos que debutan con compromiso de la región de $\mathrm{CyC}$, es responsabilidad del otorrinolaringólogo el mantener un alto índice de sospecha para poder pesquisar esta enfermedad en etapas tempranas y así entregar un buen pronóstico y opciones de curación reales a los pacientes.

\section{CONCLUSIÓN}

Nuestro estudio es una buena aproximación para conocer la realidad epidemiológica del linfoma con manifestaciones en CyC en Chile. Mostró que nuestra realidad es similar a la internacional en cuanto a la presentación y diferencias entre ambos tipos de linfoma, con algunas diferencias locales, concordantes con lo reportado en literatura nacio- nal. Creemos que los próximos estudios en el área deben enfocarse en el desarrollo de herramientas diagnósticas que permitan un diagnóstico más temprano y así mejorar la tasa de sobrevida en estos pacientes.

\section{BIBLIOGRAFÍA}

1. Jemal A, Thun MJ, Ries LA, et al. Annual report to the nation on the status of cancer, 19752005 , featuring trends in lung cancer, tobacco use, and tobacco control. J Natl Cancer Inst 2008;100(23): 1672-94.

2. Zapater e, Bagan JV, Carbonell F, Basterra J. Malignant lymphoma of the head and neck. Oral Dis 2010;16(2): 119-28.

3. Ministerio de Salud C. Guía Clínica 2008 Linfoma en Personas de 15 años y más.

4. Jaffe eS, Harris NL, Stein H, Isaacson PG. Classification of lymphoid neoplasms: the microscope as a tool for disease discovery. Blood 2008;112(12): 4384-99.

5. URquHaRt A, Berg R. Hodgkin's and nonHodgkin's lymphoma of the head and neck. Laryngoscope 2001; 111(9): 1565-9.

6. VeGA $F$, LIN $P$, MedeIRos LJ. Extranodal lymphomas of the head and neck. Ann Diagn Pathol 2005; 9(6): 340-50.

7. Weber Al, Rahemtullah A, Ferry JA. Hodgkin and non-Hodgkin lymphoma of the head and neck: clinical, pathologic, and imaging evaluation. Neuroimaging Clin N Am 2003; 13(3): 371-92.

8. Etemad-Moghadam S, Tirgary F, Keshavarz S, AlaedDINI M. Head and neck non-Hodgkin's lymphoma: a 20-year demographic study of 381 cases. Int J Oral Maxillofac Surg 2010; 39(9): 869-72.

9. Cabrera ME, García H, Lois V, et al. [Hodgkin lymphoma in Chile: experience of the national adult cancer program]. Rev Med Chil 2007; 135(3): 341-50.

10. Iyengar P, Mazloom A, Shihadeh F, Berjawi G, Dabasa B. Hodgkin lymphoma involving extranodal and nodal head and neck sites: characteristics and outcomes. Cancer 2010; 116(16): 3825-9. 
11. Lim U, Morton LM, Subar AF, et al. Alcohol, smoking, and body size in relation to incident Hodgkin's and non-Hodgkin's lymphoma risk. Am J Epidemiol 2007; 166(6): 697-708.

12. Monnereau A, Orsi L, Troussard X, et al. Cigarette smoking, alcohol drinking, and risk of lymphoid neoplasms: results of a French casecontrol study. Cancer Causes Control 2008; 19(10): 1147-60.

13. Morton LM, Zheng T, Holford TR, et al. Alcohol consumption and risk of non-Hodgkin lymphoma: a pooled analysis. Lancet Oncol 2005; 6(7): 469-76.

14. Besson H, Brennan P, Becker N, et al. Tobacco smoking, alcohol drinking and Hodgkin's lymphoma: a European multi-centre case-control study (EPILYMPH). Br J Cancer 2006; 95(3): 378-84.

15. Nieters A, Deeg E, Becker N. Tobacco and alcohol consumption and risk of lymphoma: results of a population-based case-control study in Germany. Int J Cancer 2006; 118(2): 422-30.

16. Battaglioli T, Gorini G, Costantini AS, et al. Cigarette smoking and alcohol consumption as determinants of survival in non-Hodgkin's lymphoma: a population-based study. Ann Oncol 2006; 17(8): 1283-9.

17. Canellos GP, NiedzWIeCkI D. Long-term follow-up of Hodgkin's disease trial. N Engl J Med 2002; 346(18): 1417-8.

18. Hermans J, Krol AD, van Groningen K, et al. International Prognostic Index for aggressive non-Hodgkin's lymphoma is valid for all malignancy grades. Blood 1995; 86(4): 1460-3.

19. Aanaes K, Kristensen E, Ralfkiaer eM, von Buchwald C, SPECHT L. Improved prognosis for localized malignant lymphomas of the head and neck. Acta Otolaryngol 2010; 130(5): 626-31.

Dirección: Dr. Rubén González Echeverría

Departamento de Otorrinolaringología Facultad de Medicina

Pontificia Universidad Católica de Chile

E-mail: rubensge@gmail.com 